\title{
Multiple functions integrated inside a single molecule for amplification of photodynamic therapy activity
}

Xianqing Shi ${ }^{1}$, Qichen Zhan ${ }^{1}$, Yanqing $\mathrm{Li}^{1}$, Lin Zhou ${ }^{1 *}$, Shaohua Wei ${ }^{1{ }^{2 *}}$

${ }^{1}$ College of Chemistry and Materials Science, Jiangsu Key Laboratory of Biofunctional Materials, Jiangsu Collaborative Innovation Centre of Biomedical Functional Materials, Key Laboratory of Applied Photochemistry, Nanjing Normal University, Yancheng Institute of technology, Nanjing, Jiangsu 210023, China.

${ }^{2}$ School of Chemistry and Chemical Engineering, Yancheng Institute of Technology, Yancheng, Jiangsu 224051, China.

E-mail: zhoulin@njnu.edu.cn (L. Zhou), shwei@njnu.edu.cn (S.H. Wei).

X. Shi and Q.C. Zhan contributed equally to the work.

\section{MATERIALS AND INSTRUMENTATION}

Materials. 4-Nitrophthalonitrile, p-Hydroxybenzoic acid and DIPEA, Zinc acetate were bought from J\&K Scientific (China). L-arginine ethyl ester dihydrochloride, L-lysine ethylester dihydrochloride and evans blue were bought from Aladdin (China). EDC.HCl, DBU, HOBt and ADPA were obtained from Sigma-Aldrich (USA). DMF, DMSO and other organic solvents were obtained from Sinopharm Group Co. Ltd (China) without further treatment before use. DMEM, Tyrisin, FBS were obtained from Thermo Fisher (USA). SOSG were purchased from Thermo Fisher Scientific. MTT was obtained from Amosco. DCFH-DA, DHE, Hoechst 33342, Western and IP Cell lysis buffer, Total protein extraction kit, Protein quantification kit, Ponceau S staining kit, DAF-FM DA, c-PTIO, Annexin V-FITC/PI kit and GSH and GSSG assay kit were from Beyotime (China). Tempol was purchased from Selleck.cn and L-cysteine was obtained from Huixing Biochemical Reagent Co. Ltd (China). P-gp primary antibody was obtained from Proteintech Co. Ltd (USA), Primary antibody of GAPDH, Second antibody of Peroxidase AffiniPure Goat Anti-Rabbit lgG $(H+L)$, H\&E were purchased from Yeasen Biotechnology Co. Ltd (China).

Instrumentation. IR was tested using Tensor 27 infrared spectrometer (Bruker, Germany). AN-400 MHz instrument (Bruker, Germany) was applied to ${ }^{1} \mathrm{H}$ NMR and ${ }^{13} \mathrm{C}$ NMR spectra 
detection. MS were detected using MS-ESI (Agilent, USA) or MS-MALDI-TOF spectrometer (Bruker, Germany) in accordance with requirements. UV-vis spectrum was detected using a Cary 50 spectrophotometer (Varian, USA). Fluorescence spectra was recorded by Cary Eclipse fluorescence spectrophotometer (Varian, USA). Cell viability was carried out using a microplate reader (Thermo, USA). The CLSM (Nikon Japan) was used for obtaining the fluorescence microscopy images. FCM measurement were monitored via XL flow cytometer (Beckman, USA). The electrophoresis apparatus (DYY-6C) was used to operate with immunoprotein imprinting. ECL luminescence images were observed by automatic chemiluminescence image analyzer (Tanon-5200). In vivo fluorescence images were obtained by IVIS Lumina K (Series III).
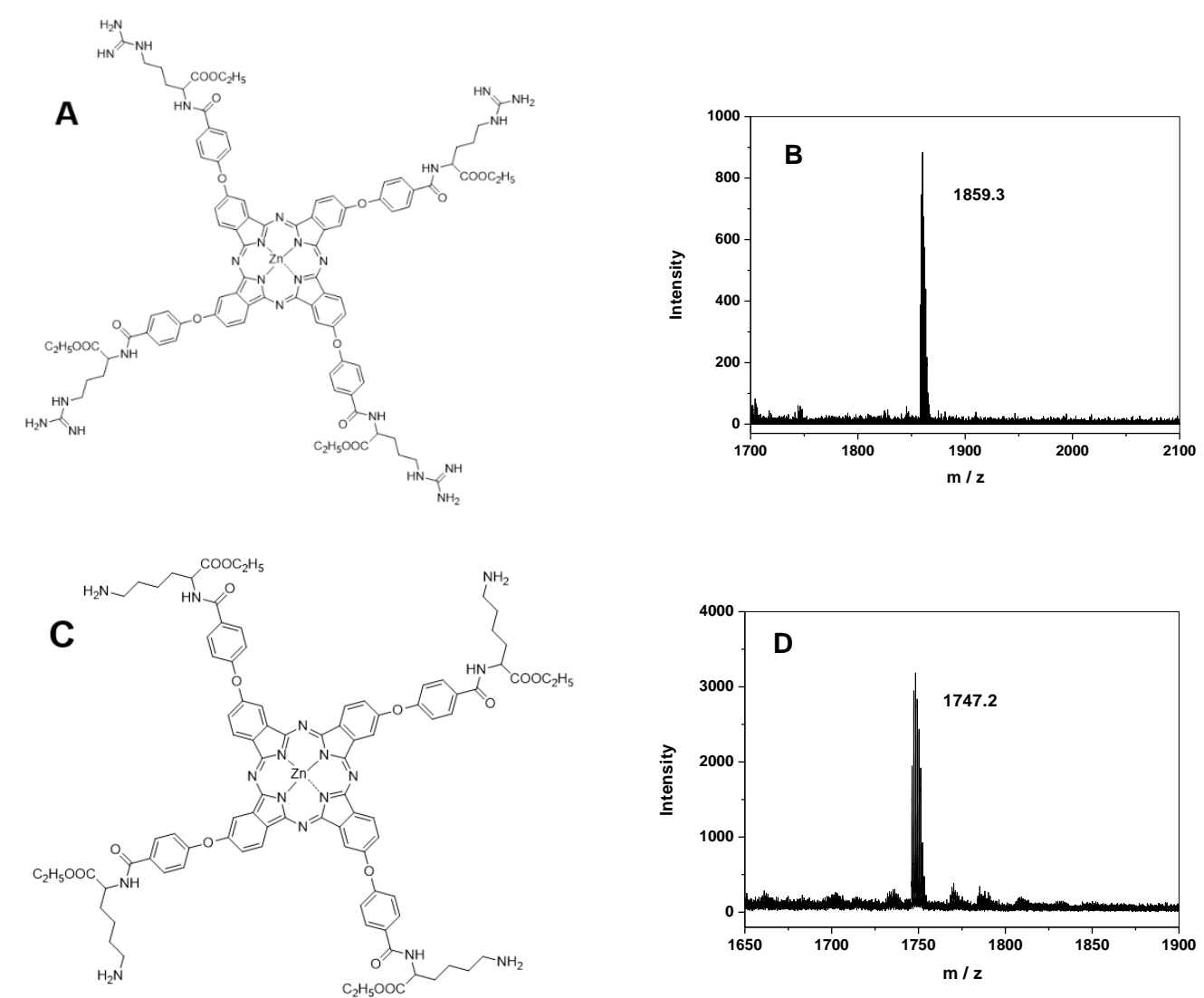

Figure S1. The structures of $\operatorname{Arg}-\mathrm{ZnPc}(\mathrm{A})$ and $\mathrm{Lys}-\mathrm{ZnPc}(\mathrm{C})$ and the MS-MALDI-TOF spectrometer of $\mathrm{Arg}-\mathrm{ZnPc}(\mathrm{B})$ and Lys-ZnPc (D) (Only the major $\mathrm{C}_{4 \mathrm{~h}}$ isomer is shown for Arg-ZnPc and Lys-ZnPc, which likely contains other isomers).

Arg-ZnPc and Lys-ZnPc were dissolved in N,N-dimethylformamide (DMF) with high concentration (2 $\mathrm{mM})$ as stock-solutions. We detected the UV-Vis absorbance spectra from 
500 to $800 \mathrm{~nm}$ of $\mathrm{Arg}-\mathrm{ZnPc}$ and Lys-ZnPc in aqueous solutions $\left(3 \mathrm{~mL} \mathrm{H}_{2} \mathrm{O}\right.$ with various volume of above stock-solutions, volume fraction of DMF in $\mathrm{H}_{2} \mathrm{O}$ were $0.3 \%, 0.6 \%, 0.9 \%, 1.2 \%, 1.5 \%$, $1.8 \%$ and $2.1 \%$ ) with different $\mathrm{ZnPc}$ concentration $(2,4,6,8,10,12$ and $14 \mu \mathrm{M})$. And the fitting results match the Lambert-Beer law. The molar extinction coefficients $(\varepsilon)$ at various wavelength were calculated according to the following Equation,

$$
A=\varepsilon c b
$$

Where $A$ is the measured absorbance, $b$ is the path length, and $c$ is the analyte concentration. And we obtained molar extinction coefficients at various wavelength $\left(\varepsilon_{\text {at aggregator, }}\right.$,

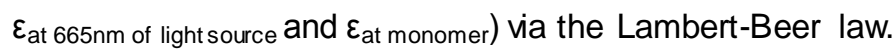

Table S1. Molar extinction coefficients at various wavelength $\left(\varepsilon_{\text {at }} 632 \mathrm{~mm}\right.$ of aggregator, $\varepsilon_{\text {at }} 665 \mathrm{~mm}$ of light source and $\varepsilon_{\text {at }} 674 \mathrm{~nm}$ of monomer, Table S1) of Arg-ZnPc and Lys-ZNPc.

\begin{tabular}{|c|c|c|c|}
\hline $\mathrm{ZnPc}$ & $\varepsilon_{\text {at } 632 \mathrm{~nm} \text { of aggregator }}\left(\mathrm{M}^{-1} \mathrm{~cm}^{-1}\right)$ & $\varepsilon_{\text {at } 665 n m}$ of light source $\left(\mathrm{M}^{-1} \mathrm{~cm}^{-1}\right)$ & $\varepsilon_{\text {at } 674 \mathrm{~nm} \text { of monomer }}\left(\mathrm{M}^{-1} \mathrm{~cm}^{-1}\right)$ \\
\hline Arg-ZnPc & $2.872 \times 10^{4}$ & $1.810 \times 10^{4}$ & $1.608 \times 10^{4}$ \\
\hline Lys-ZnPc & $2.235 \times 10^{4}$ & $2.443 \times 10^{4}$ & $2.576 \times 10^{4}$ \\
\hline
\end{tabular}
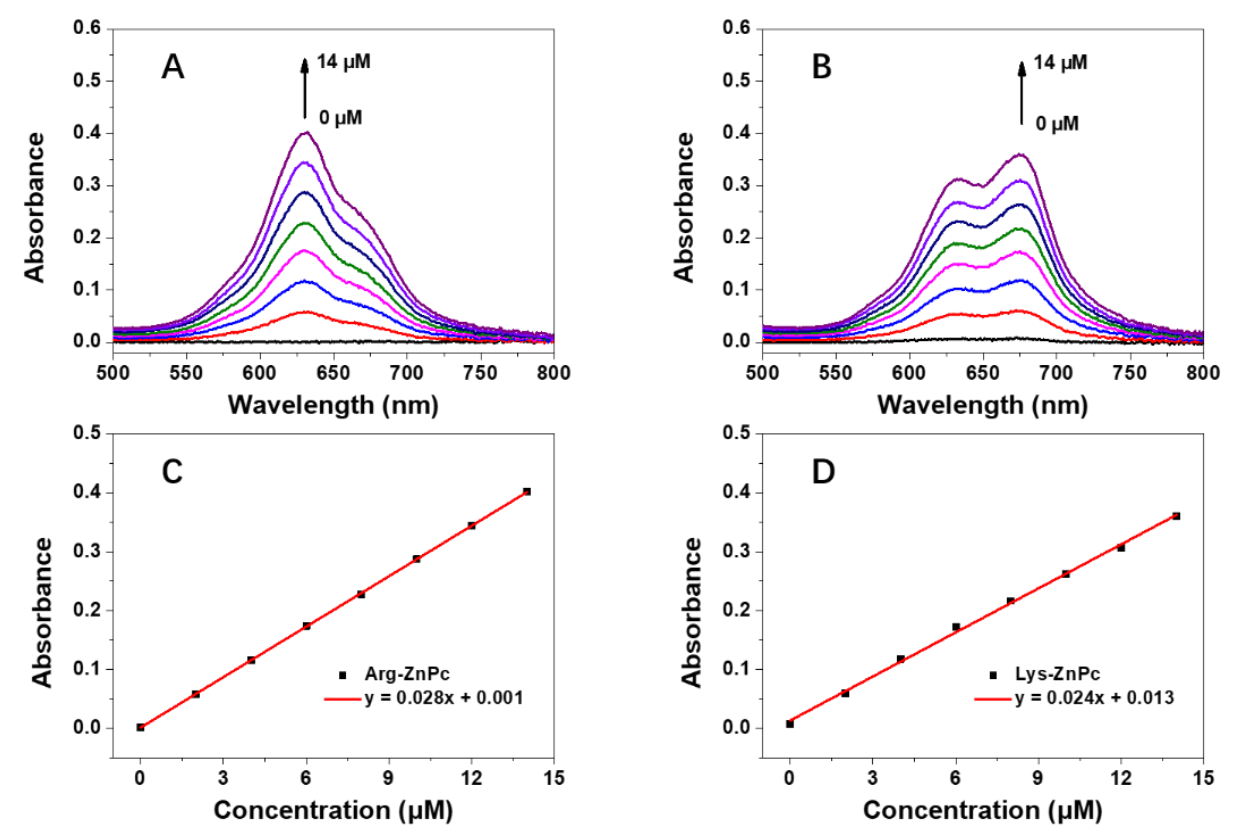

Figure S2. The molar extinction coefficient $(\varepsilon)$ of Arg-ZnPc and Lys-ZnPc by UV-Vis spectrum. 


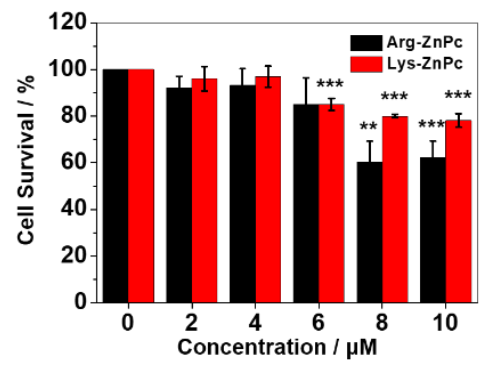

Figure S3. Dark toxicity comparison of Arg-ZnPc and Lys-ZnPc with different concentration (the value of ${ }^{* *} p<0.01$ and ${ }^{* * *} p<0.001$ were the drugs treated groups versus control).

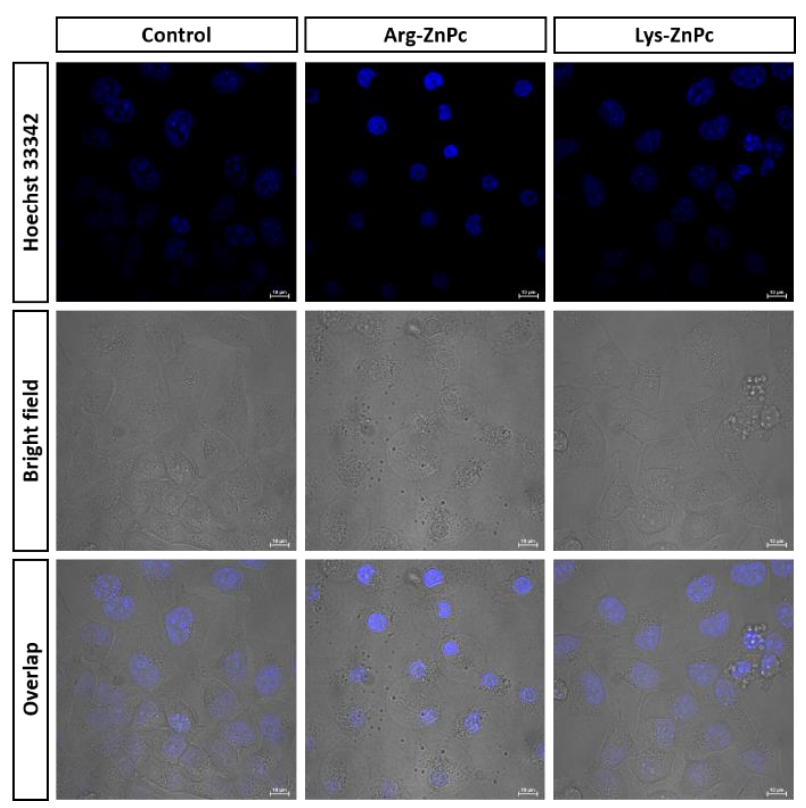

Figure S4. Hoechst 33342 staining with control, Arg-ZnPc and Lys-ZnPc after irradiation (Bar $=10 \mu \mathrm{m})$.
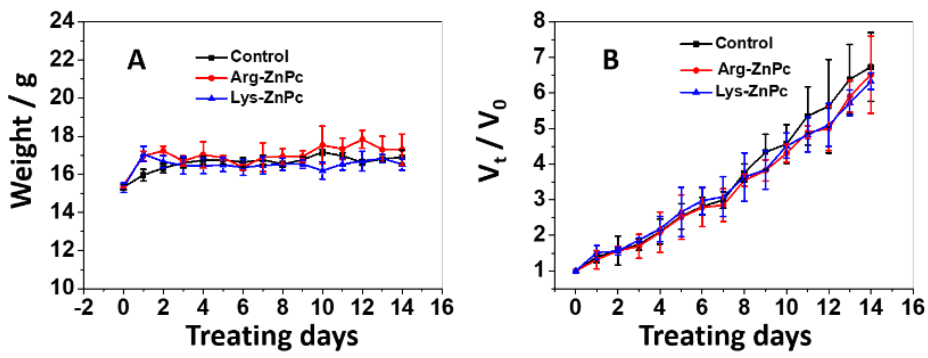

Figure S5. The weight (A) and relative tumor volume (B) changes of the mice during 14 days' treatment by various drugs without light irradiation at tumor tissue. 


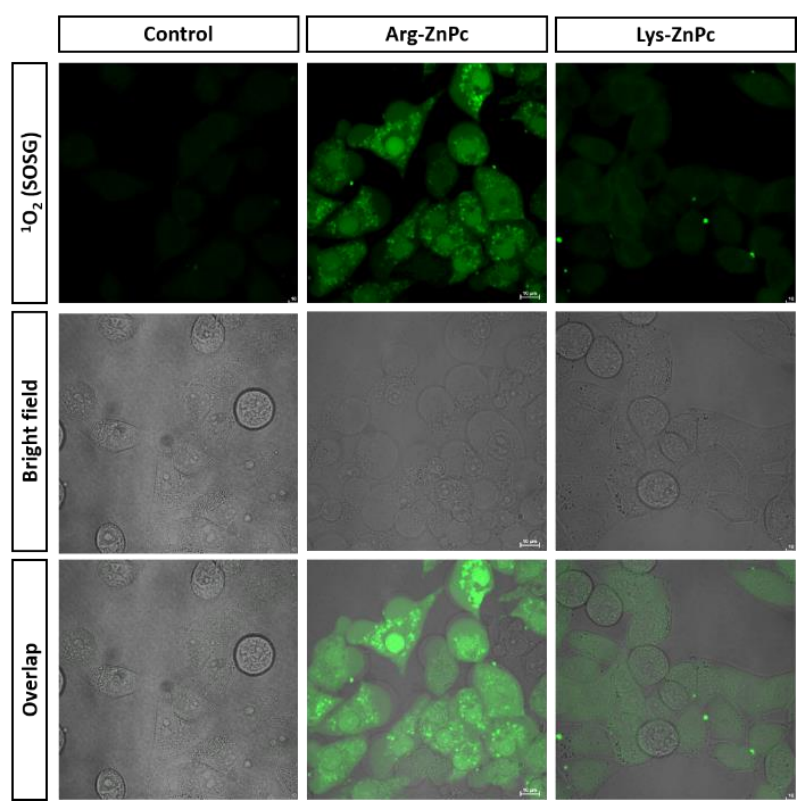

Figure S6. Fluorescence images comparison of intracellular ${ }^{1} \mathrm{O}_{2}$ was detected by SOSG after irradiation $(B a r=10 \mu \mathrm{m})$.

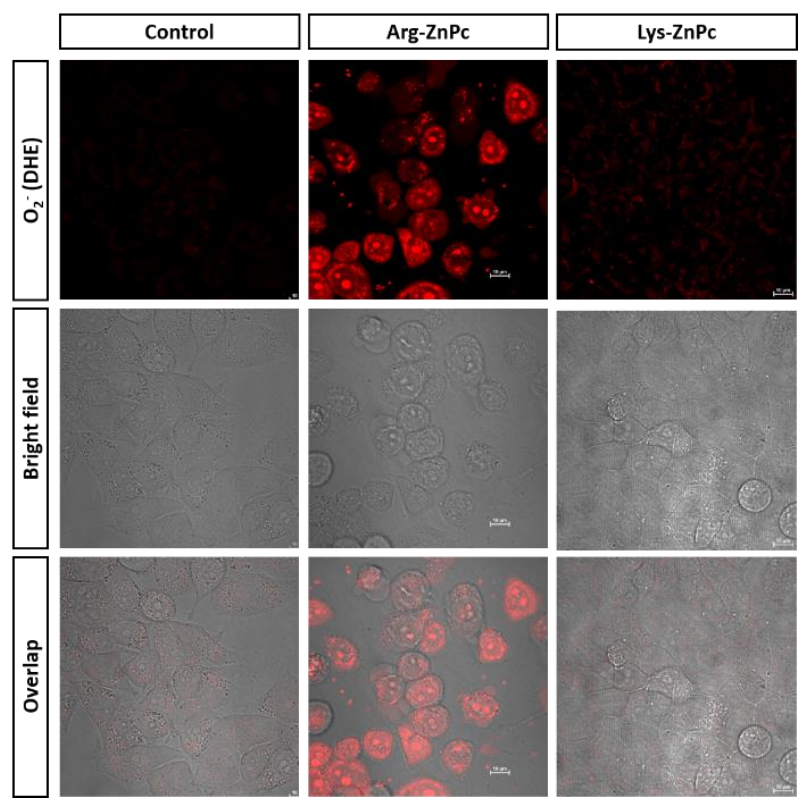

Figure S7. Fluorescence images comparison of intrac ellular $\mathrm{O}_{2}{ }^{--}$was detected by DHE after irradiation $($ Bar $=10 \mu \mathrm{m})$. 


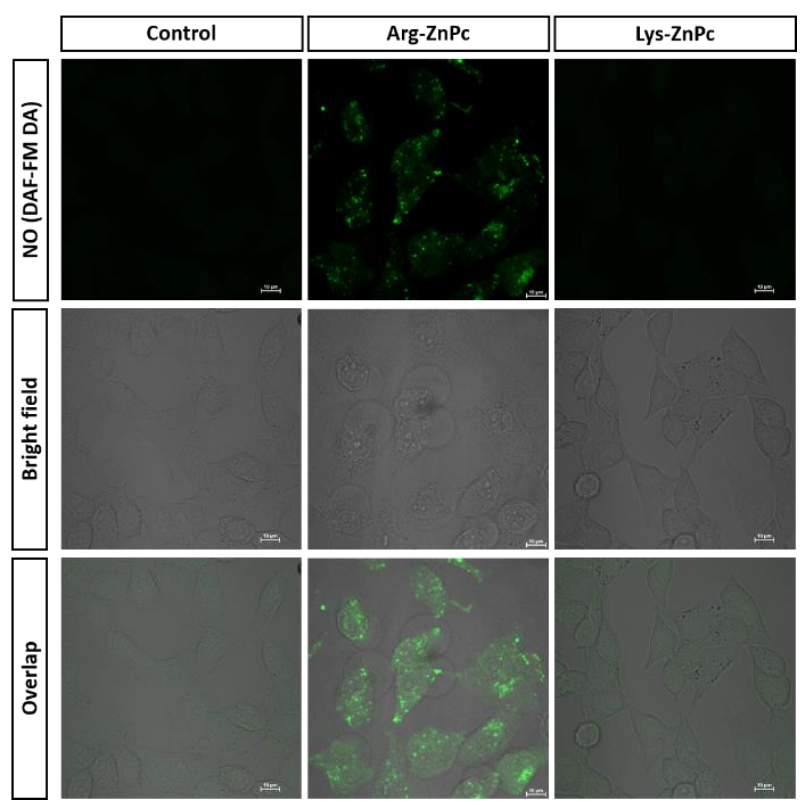

Figure S8. Fluorescence images comparison of intracellular NO was detected by DAF-FM DA after irradiation $($ Bar $=10 \mu \mathrm{m})$.

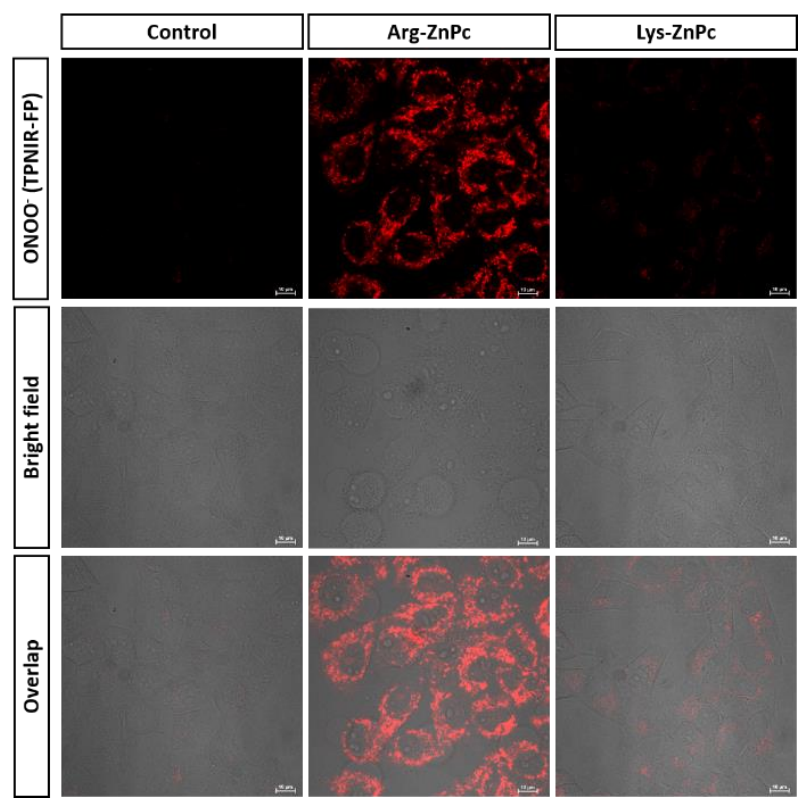

Figure S9. Fluorescence images comparison of intracellular $\mathrm{ONOO}^{-}$was detected by TPNIR-FP after irradiation $($ Bar $=10 \mu \mathrm{m})$. 


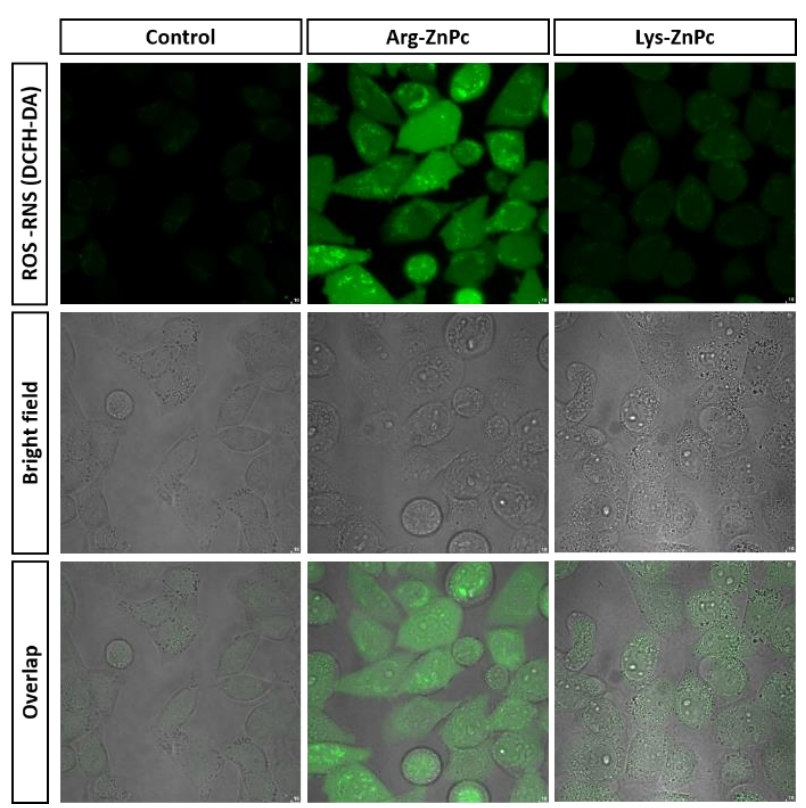

Figure S10. Fluorescence images comparison of intracellular ROS+RNS was detected by DCFH-DA after irradiation $(B a r=10 \mu \mathrm{m})$.

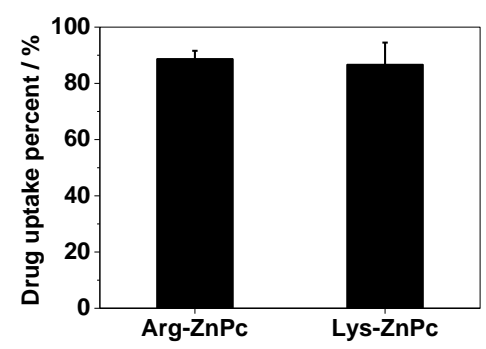

Figure S11. The detection of drug efflux effect by Arg-ZnPc or Lys-ZnPc at $48 \mathrm{~h}$ drug incubation.

The ROS/RNS probe experiments were validated using their specific quenchers. C-PTIO ${ }^{1}$, ADPA $^{2}$, tempol ${ }^{3}$, L-cysteine ${ }^{4}$ were selected as the scavenger of $\mathrm{NO},{ }^{1} \mathrm{O}_{2}, \mathrm{O}_{2}{ }^{-*}$, ONOO", respectively. The above results were listed as follows. 

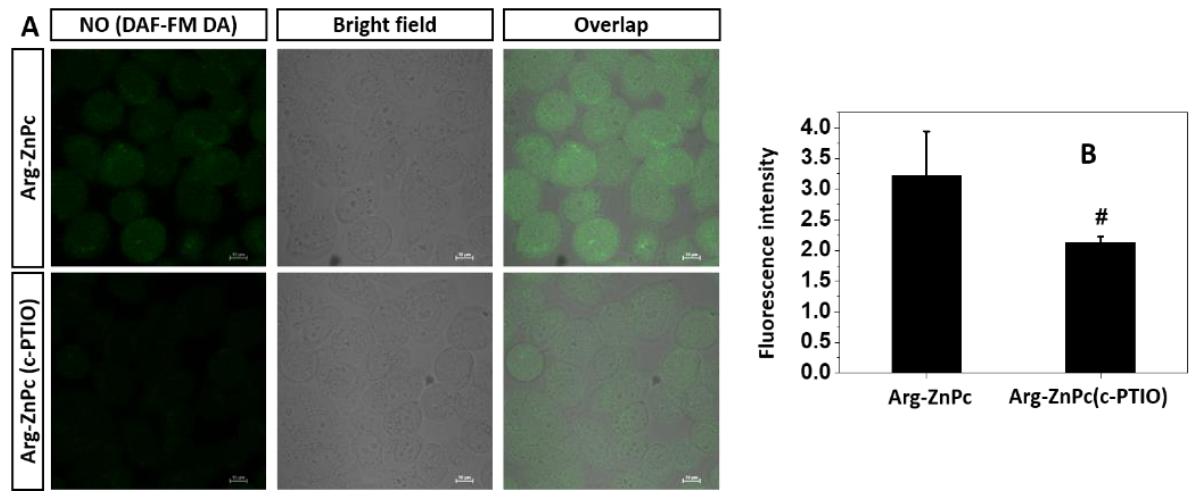

Figure S12. Fluorescence images (A) and intensity (B) of intracellular NO was detected by DAF-FM DA after adding c-PTIO (Bar $=10 \mu \mathrm{m})$.
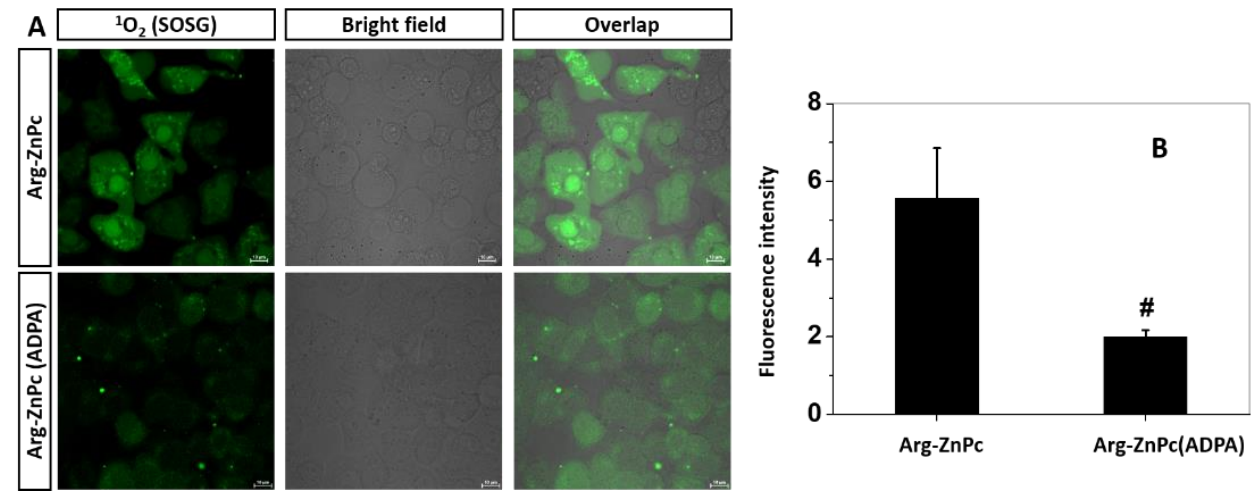

Figure S13. SOSG probe fluorescence signal (A) and intensity (B) changing of $\mathrm{Arg}-\mathrm{ZnPc}$ and light treated cells with or without ${ }^{1} \mathrm{O}_{2}$ specific quencher $\operatorname{ADPA}(\mathrm{Bar}=10 \mu \mathrm{m})$.
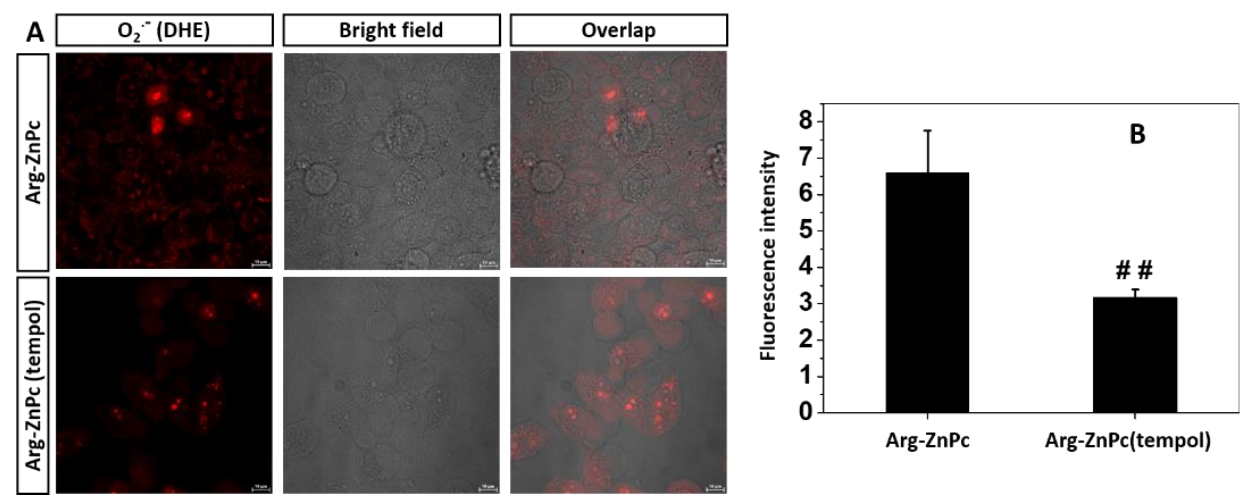

Figure S14. DHE probe fluorescence signal (A) and intensity (B) changing of $\mathrm{Arg}-\mathrm{ZnPc}$ and light treated cells with or without $\mathrm{O}_{2}{ }^{--}$specific quencher tempol $(\mathrm{Bar}=10 \mu \mathrm{m})$. 

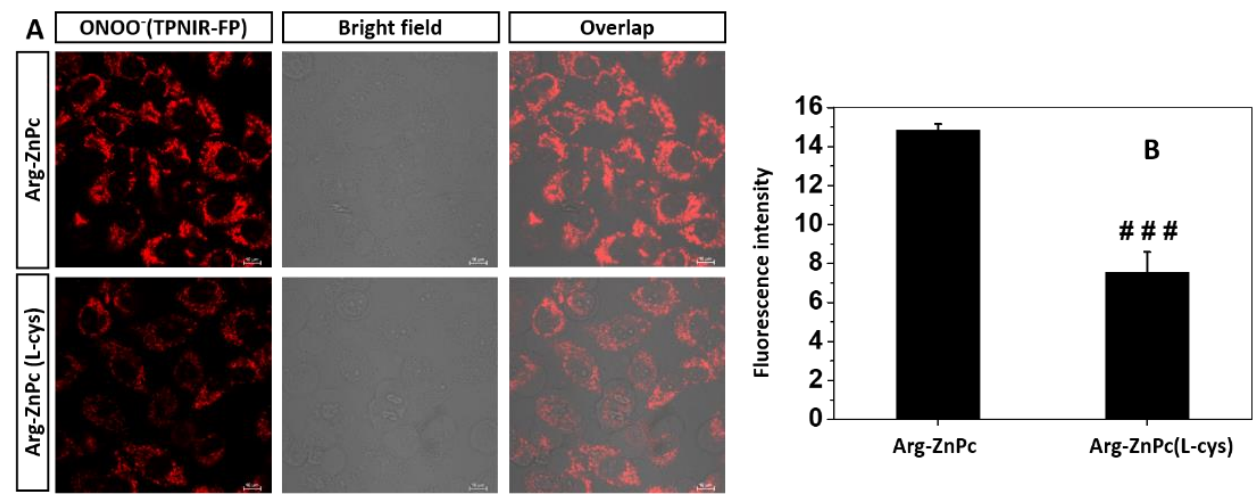

Figure S15. TPNIR-FP probe fluorescence signal (A) and intensity (B) changing of Arg-ZnPc and light treated cells with or without $\mathrm{ONOO}^{-}$specific quencher L-cysteine $(\mathrm{Bar}=10 \mu \mathrm{m})$.

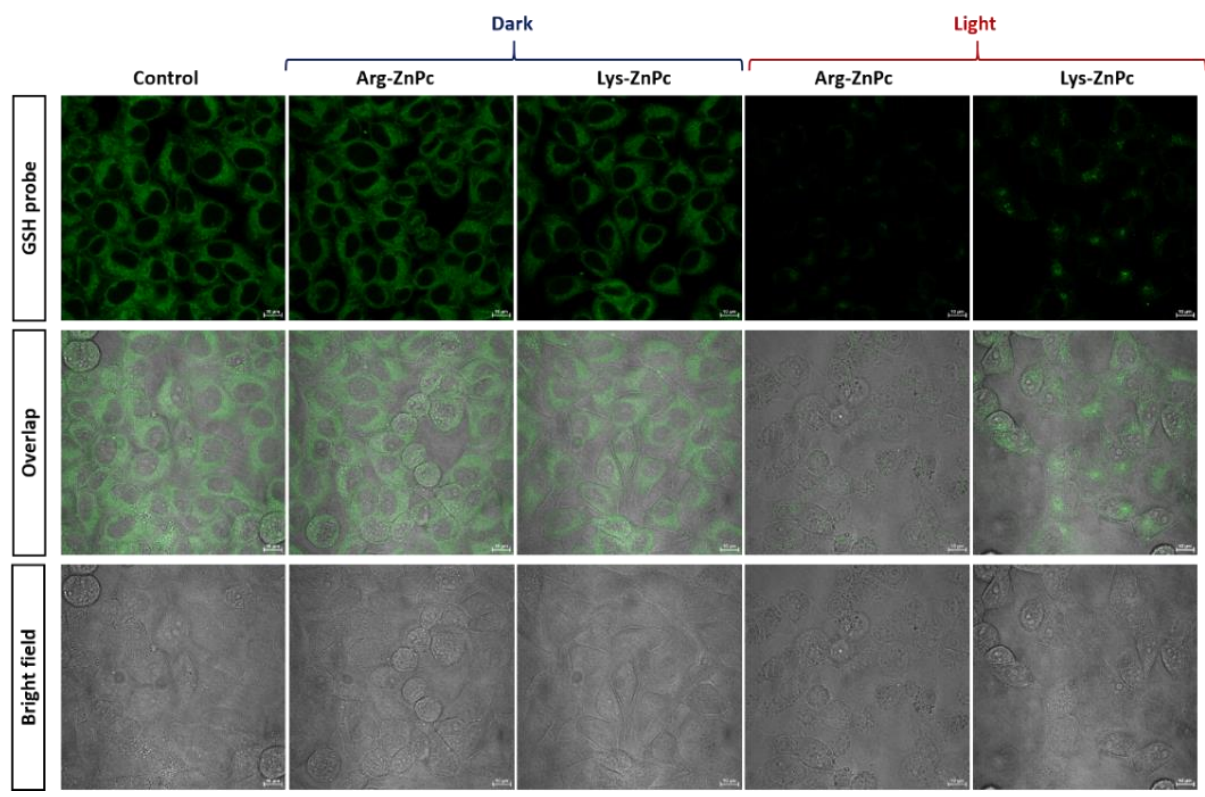

Figure S16. Fluorescence images comparison of intracellular GSH concentration was detected after treating $\mathrm{Arg}-\mathrm{ZnPc}$ and $\mathrm{Lys}-\mathrm{ZnPc}$ under dark and light irradiation (Bar $=10 \mu \mathrm{m})$. 


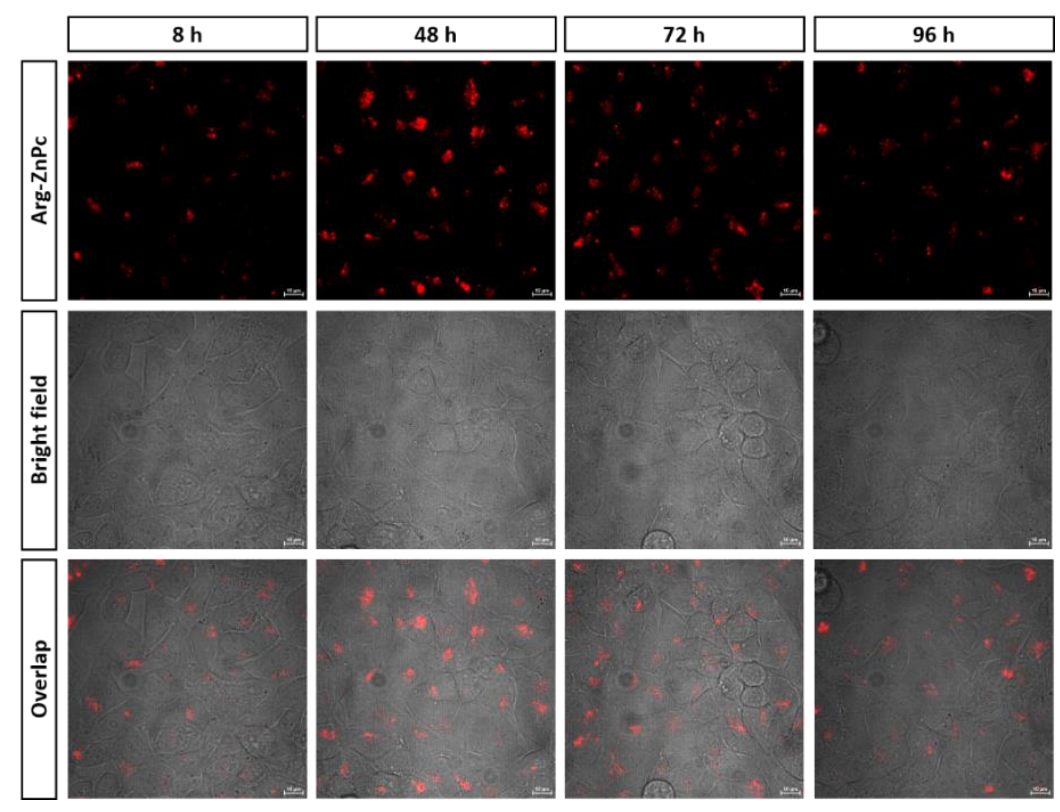

Figure S17. The detection of $\mathrm{Arg}-\mathrm{ZnPc}$ efflux effect with the prolonged incubation time $(\mathrm{Bar}=$ $10 \mu \mathrm{m})$.

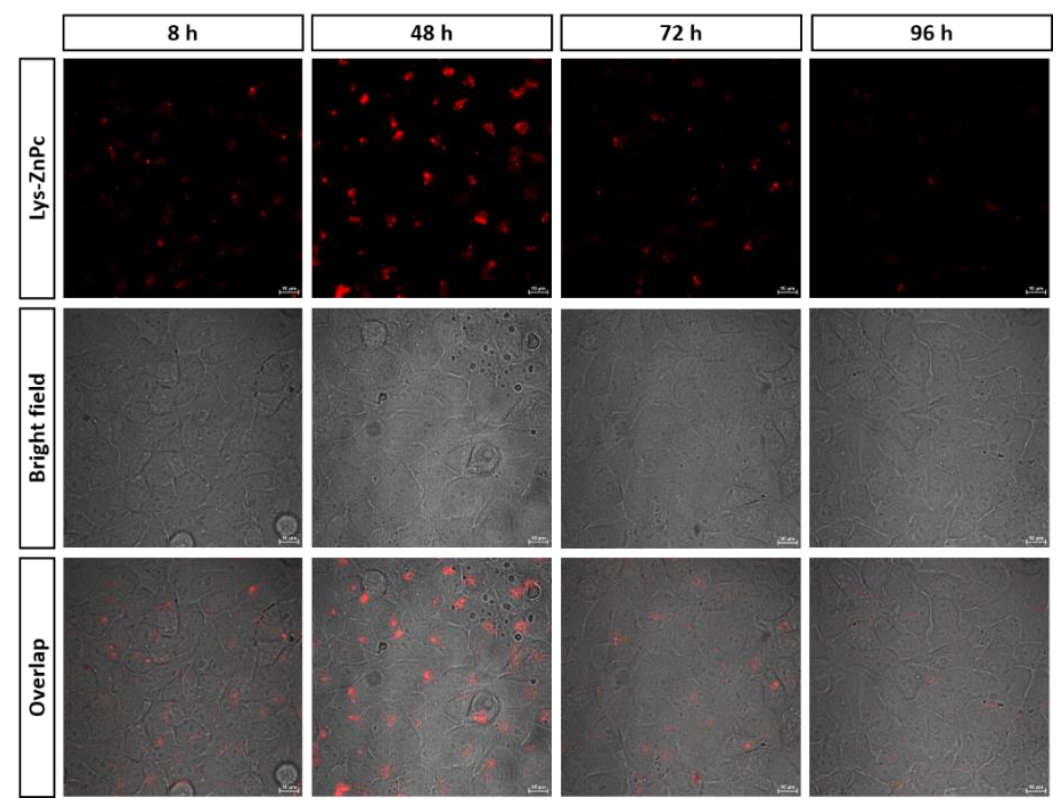

Figure S18. The detection of Lys-ZnPc efflux effect with the prolonged incubation time (Bar= $10 \mu \mathrm{m})$. 


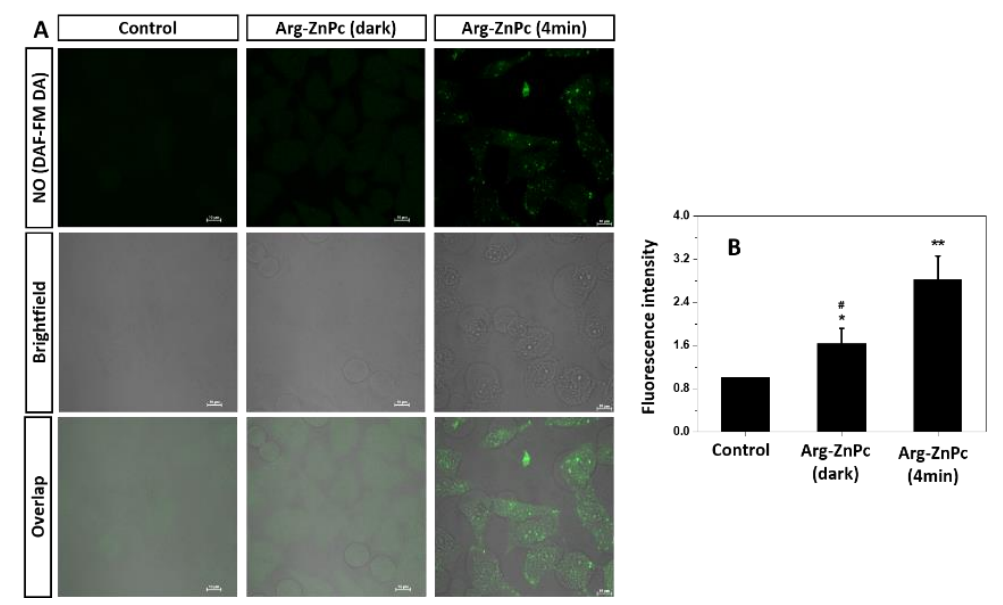

Figure S19. Fluorescence images (A) and intensity (B) of NO generation, detecting by DAF-FM DA, in Arg-ZnPc treated cells without or with 4 min irradiation time $(\mathrm{Bar}=10 \mu \mathrm{m})$.

\section{REFERENCES}

1. He, H.; Feng, Y. S.; Zang, L. H.; Liu, W. W.; Ding, L. Q.; Chen, L. X; Kang, N.; Hayashi, T.; Tashiro, S.; Onodera, S.; Qiu, F.; Ikejima, T. Nitric oxide induces apoptosis and autophagy; autophagy down-regulates NO synthesis in physalin A-treated A375-S2 human melanoma cells. Food Chem. Toxicol. 2014, 71, 128-135.

2. He, J.; Wang, Y.; Missinato, M. A.; Onuoha, E.; Perkins, L. A.; Watkins, S. C.; St Croix, C. M.; Tsang, M.; Bruchez, M. P. A genetically targetable near-infrared photosensitizer. Nat. Methods 2016, 13, 263-268.

3. Park. Tiron, a ROS scavenger, protects human lung cancer Calu-6 cells against antimycin A-induced cell death. Oncol. Rep. 2009, 21, 253-261.

4. Phelps, D. T.; Ferro, T. J.; Higgins, P. J.; Shankar, R.; Parker, D. M.; Johnson, A. TNF-alpha induces peroxynitrite-mediated depletion of lung endothelial glutathione via protein kinase C. American J. Phy.-Lung Cellular and Molecular Phy. 1995, 269, 551-559. 